\title{
Growth of a sandpile around an obstacle
}

\author{
Mikhail Feldman \\ Department of Mathematics, Northwestern University, 2033 Sheridan Road, Evanston, IL 60208-2730
}

\section{Introduction}

The geometry of optimal Monge-Kantorovich mass transfer in $\mathbf{R}^{n}$ for various cost functions has been studied recently by many authors, see survey $[\mathrm{E}]$ and references therein.

A natural next step is to describe the geometry of optimal Monge-Kantorovich mass transfer around an obstacle. Let $\Omega \subset \mathbf{R}^{n}$ be a strictly convex open bounded set with smooth boundary. Let $\Lambda=\mathbf{R}^{n} \backslash \Omega$. The set $\Omega$ will be regarded as an obstacle. The problem is to understand the geometry of the optimal mass transfer in the domain $\Lambda$. In this paper we consider one particular example, which can be interpreted as a model of growth of a sandpile around an obstacle.

A model of growth of a sandpile fed by point sources was proposed by G. Aronsson in [Ar72]. Aronsson considers two cases. The first case is growth of a sandpile on a flat horizontal surface without obstacle, i.e., $\Lambda=\mathbf{R}^{n}$. The second case is growth of a sandpile on a flat horizontal surface in the presence of an obstacle which has the form of vertical cylinder $\Omega \times \mathbf{R}^{1}$, i.e., $\Lambda \neq \mathbf{R}^{n}$. According to the Aronsson's model in the case $\Lambda=\mathbf{R}^{n}$ a sandpile has the form of interpenetrating circular cones centered at the locations of sources. The slope of cones is defined by a given critical value. The cones grow, the heights of cones are functions of time and satisfy a certain system of ODE. In the presence of an obstacle the model is similar, with the main difference that the shape of "cones" depends on $\Lambda$.

In the case $\Lambda=\mathbf{R}^{n}$, G. Aronsson, L. C. Evans, Y. Wu [AEW96] show the relation of the model of [Ar72] to an evolution governed by a subdifferential of a convex functional. This in particular extends the model of [Ar72] to the case of sand sources more general than the point sources. The connection between such variational problems and Monge-Kantorovich mass transfer was observed by L. C. Evans and W. Gangbo in [EGan].

In this paper we extend the variational model of [AEW96] to the case when there is an obstacle $\Omega$, and so $\Lambda \neq \mathbf{R}^{n}$. The main physical assumption in the model is that the sandpile is stable only if its slope is less than or equal to one. We show that for a single point sand source our model admits the solution given by the Aronsson's model [Ar72], and this solution is unique. The main question we answer is the following: How does sand move around the obstacle, i.e., what is the geometry of mass transfer in the presence of an obstacle? We consider the specific case of a single point source in the dimension two since in this case we have an explicit solution of the model. We show that each sand particle moves instantaneously from the source point to its resting place along the shortest path (note that in the presence of an obstacle the shortest path is not necessarily a segment). It is important that this principle is not an a priori assumption. We derive it from our variational model. We also compute the rate of mass transport through each point. We obtain these results by constructing the mass transport density. Such function was first introduced in [EGan] in the case $\Lambda=\mathbf{R}^{n}$, and used for analysis of variational evolution problems in [EFG97], [F97]. 
In our case, due to the presence of an obstacle, the mass transport density is a measure which has concentrations on the subsets of lower dimension.

A more general case of mass transfer around an obstacle (i.e., higher dimension, and the source and destination to be more general measures) will be considered in a forthcoming paper.

The paper is organized as following. In Section 2 we define our model and prove some basic facts. In Section 3 we give the Monge-Kantorovich mass transfer interpretation of the model. In Sections 4 and 5 we consider a single point source solution of the model in the dimension two. In Section 4 we define transport rays and describe their structure. Section 5 is the main part of the paper. We construct the mass transport density, prove its uniqueness, and describe the geometry of mass transfer.

Remark 1 A more general model of sandpile growth over given landscape was proposed by

L. Prigozhin [P96].

Calculations related to ones in Section 5 can be found in [J96], [F97].

\section{Sandpile growth model}

Let $\Omega \subset \mathbf{R}^{n}$ be a strictly convex open bounded set with smooth boundary, and $\Lambda=\mathbf{R}^{n} \backslash \Omega$.

Let $x, y \in \Lambda$. Denote $d_{\Lambda}(x, y)$ the distance between $x$ and $y$ in $\Lambda . d_{\Lambda}(x, y)$ is defined as infimum of the lengths of smooth paths that lie within $\Lambda$ and connect $x$ and $y$, i.e.

$$
\begin{gathered}
d_{\Lambda}(x, y)=\inf \left\{\int_{0}^{1}|\dot{\gamma}(s)| d s \quad \mid \gamma \in C^{1}\left([0,1] ; \mathbf{R}^{n}\right), \gamma(s) \in \Lambda \quad \text { for all } s \in[0,1]\right. \\
\gamma(0)=x, \quad \gamma(1)=y\}
\end{gathered}
$$

Clearly,

$$
d_{\Lambda}(x, y) \leq C|x-y|
$$

Let $I_{\Lambda}^{\infty}: L^{2}(\Lambda) \rightarrow \mathbf{R}^{1} \cup\{\infty\}$ be defined by

$$
I_{\Lambda}^{\infty}[v]= \begin{cases}0 & \text { if } v \in L^{2}(\Lambda) \cap \operatorname{Lip}(\Lambda), \quad|D v| \leq 1 \text { a.e. in } \Lambda \\ +\infty & \text { otherwise, }\end{cases}
$$

where $\operatorname{Lip}(\Lambda)$ is the set of Lipschitz continuous functions. Thus we have $I_{\Lambda}^{\infty}[v]=0$ if and only if $v \in L^{2}(\Lambda)$ and

$$
|v(x)-v(y)| \leq d_{\Lambda}(x, y) \quad \text { for any } \quad x, y \in \Lambda .
$$

Definition 2 A function $v: \Lambda \rightarrow \mathbf{R}^{1}$ is Lipschitz on $\Lambda$ with Lipschitz constant at most one if (2) holds. We denote $\operatorname{Lip}_{1}(\Lambda)$ the set of functions Lipschitz on $\Lambda$ with Lipschitz constant at most one.

We consider the evolution problem

$$
\begin{cases}f-\partial_{t} u \in \partial I_{\Lambda}^{\infty}[u] & \text { for a.e. } t>0 \\ u=g & \text { on } \Lambda \times\{t=0\}\end{cases}
$$

where $g \in L^{2}(\Lambda)$ is a nonnegative Lipschitz function satisfying

$$
|D g| \leq 1 \quad \text { almost everywhere in } \Lambda,
$$


or equivalently $g \in \operatorname{Lip}_{1}(\Lambda) \cap L^{2}(\Lambda)$. We interpret the problem (3) as a model of sandpile growth in $\Lambda$ as following. The function $u(\cdot, t)$ is the height of a pile of sand at time $t$. The term $f$ describes the sand sources. Specifically, $f(x, t)$ is the rate at which sand is added at the location $x \in \Lambda$ at time $t$. We assume that $f$ is nonnegative. The main physical assumption encoded into this model is that a sandpile is stable if and only if the slope is less than or equal to one. The model describes the growth of an initially stable sandpile on the $n$-dimensional flat surface with the cylindric obstacle $\Omega \times[0, \infty)$.

The problem considered in [AEW96] has the form of (3) with the functional $I^{\infty}$ defined on $\mathbf{R}^{n}$ instead of $\Lambda$. The new feature in our work is the presence of an obstacle.

Remark 3 Similar to [AEW96], we interpret the differential inclusion (3) as following. The inclusion (3) implies that for a.e. $t>0$, for each $v \in L^{2}(\Lambda)$

$$
I_{\Lambda}^{\infty}[v] \geq I_{\Lambda}^{\infty}[u]+\int_{\Lambda}\left(f(x, t)-\partial_{t} u(x, t)\right)(v(x)-u(x, t)) d x .
$$

As $I_{\Lambda}^{\infty}[v]=+\infty$ if $v \notin \operatorname{Lip}_{1}(\Lambda)$, it is enough to consider only $v \in \operatorname{Lip}_{1}(\Lambda)$. But then $I_{\Lambda}^{\infty}[v]=0$. Thus (3) means that for a.e. $t>0$ there holds $u(\cdot, t) \in \operatorname{Lip}_{1}(\Lambda) \cap L^{2}(\Lambda)$ and for every $v \in \operatorname{Lip}_{1}(\Lambda) \cap L^{2}(\Lambda)$

$$
\int_{\Lambda}\left(f(x, t)-\partial_{t} u(x, t)\right)(v(x)-u(x, t)) d x \leq 0 .
$$

Since $u(\cdot, t)$ and $v$ are Lipschitz functions, the left-hand side of (4) is defined for quite general $f$. In particular consider

$$
f(x, t)=f_{0}(t) \delta_{p}(x),
$$

where $p \in \Lambda^{0}\left(\Lambda^{0}\right.$ is the interior of $\left.\Lambda\right), \delta_{p}$ is a Dirac mass at the point $p$, and $f_{0}(t) \geq 0$. This $f(x, t)$ represents a single point source at the location $p$. For such $f$ we interpret the inequality (4) as

$$
f_{0}(t)[v(p)-u(p, t)] \leq \int_{\Lambda} \partial_{t} u(x, t)[v(x)-u(x, t)] d x
$$

In the remaining part of this section we show that in the case of the point source (5) our model has the solution given by the ODE model of [Ar72]. More precisely, we show that the solution of (3) with right-hand side (5) is

$$
u(x, t)=\max \left\{g(x), z(t)-d_{\Lambda}(x, p)\right\},
$$

where $z(t)$ is defined as following. Let

$$
D(t)=\left\{\quad x \in \Lambda \quad \mid z(t)-d_{\Lambda}(x, p)>g(x)\right\} .
$$

The function $z(t)$ is the solution of

$$
\left\{\begin{array}{l}
\frac{d z}{d t}(t)=\frac{f_{0}(t)}{|D(t)|}, \quad t>0 \\
z(0)=g(p) .
\end{array}\right.
$$

Note that at time $t$ the sandpile occupies the region

$$
\left\{0 \leq x_{n+1} \leq u(x, t) \mid x \in \Lambda\right\} \subset \Lambda \times[0, \infty) .
$$


If $u(x, t)$ is given by (7) in the case $\Lambda=\mathbf{R}^{n}$ and $g \equiv 0$, then the region (10) has the form of the spherical cone centered at $p$, with slope one and height $z(t)$. Thus we call the solution (7) a growing cone, although in the case $\Lambda \neq \mathbf{R}^{n}$ the region (10) is not a spherical cone in general.

We study first the problem (9). The techniques of [AEW96] can be used to obtain

Lemma 4 Let $f_{0}(t)$ be a positive Lipschitz function on $[0, \infty)$. Let $g(t)$ be nonnegative and satisfy

$$
\text { ess } \sup _{\mathbf{R}^{n}}|D g|<1 .
$$

Let $p \in \Lambda, T>0$. Then there exists a unique function $z(t) \in C^{1}((0, T]) \cap C^{0, \frac{1}{n+1}}([0, T])$ satisfying (9).

Proof. By assumption $\Omega$ is a bounded, smooth, convex set and $\Lambda=\mathbf{R}^{n} \backslash \Omega$. It follows that there exist constants $C>c>0$ depending only on $n, \Omega$ such that for any $r>0, p \in \Lambda$

$$
C r^{n} \geq\left|\left\{x \in \Lambda \mid d_{\Lambda}(x, p)<r\right\}\right| \geq c r^{n} .
$$

Now the proof of existence follows the proof of Theorem 4.1 of [AEW96], steps 1-3, 5, 6 . The differences in the argument are only notational, i.e., the Euclidean distance should be replaced by $d_{\Lambda}(\cdot, \cdot)$. In our case this argument provides the solution on the time interval $(0, \infty)$ because we have a single point source.

Uniqueness follows from the argument of [AEW96], Theorem 4.1, step 8.

Now we prove that in the case of a single point source (5) our model admits the solution (7). The argument follows closely the proof of Theorem 5.1 of [AEW96].

Proposition 5 Let $f$ be defined by (5). Let $f_{0}, g$ satisfy assumptions of Lemma 4. Let $z(t)$ be the solution of (9). Then the function (7) is a unique solution of the problem (3) in the sense of Remark 3. That is, for a. e. $t>0$, for all $v \in \operatorname{Lip}_{1}(\Lambda) \cap L^{2}(\Lambda)$ the inequality (6) holds.

Proof. Since $\Omega$ is convex and $\partial \Omega$ is smooth and since $g$ satisfies (11) it follows that $|\partial D(t)|=0$ for $t>0$. Thus for each $t>0, \partial_{t} u$ exists a.e. and

$$
\partial_{t} u(x, t)= \begin{cases}\frac{d z}{d t}(t) & \text { on }(D(t))^{0}, \\ 0 & \text { on } \Lambda \backslash \overline{D(t)} .\end{cases}
$$

We show that (6) is satisfied for every $t>0$. Substituting (12) into the right-hand side of (6) and rearranging, we see that we have to prove that

$$
\frac{1}{|D(t)|} \int_{D(t)}[v(p)-v(x)] d x \leq \frac{1}{|D(t)|} \int_{D(t)}[u(p, t)-u(x, t)] d x
$$

for every $t>0, v \in \operatorname{Lip}_{1}(\Lambda) \cap L^{2}(\Lambda)$. But this is true since

$$
|v(p)-v(x)| \leq d_{\Lambda}(p, x)=u(p, t)-u(x, t)
$$

for any $x \in D(t)$.

Uniqueness can be proved like in Theorem 3.3 of [AEW96]. 


\section{Monge-Kantorovich mass transfer interpretation of the model}

We review in this section some basic facts of the Monge-Kantorovich theory (following [R84], [E], [EGan]) and show the relation to the model (3). Since in Section 5 we consider mass transfer in the domain $\Lambda$, we modify the definitions to cover this case.

Let $\Lambda \subset \mathbf{R}^{n}$ be a domain with smooth boundary. Let $f^{+}, f^{-}: \Lambda \rightarrow \mathbf{R}^{1}$ be two nonnegative summable functions with compact supports and suppose that the following mass balance relation is satisfied

$$
\int_{\Lambda} f^{+} d x=\int_{\Lambda} f^{-} d y
$$

Define the measures $\mu^{+}=f^{+}(x) d x$ and $\mu^{-}=f^{-}(y) d y$ on $\Lambda$. We say that a one-to-one map $s: \Lambda \rightarrow \Lambda$ transfers $\mu^{+}$onto $\mu^{-}$if for any $h \in C(\Lambda)$

$$
\int_{\Lambda} h(s(x)) f^{+}(x) d x=\int_{\Lambda} h(y) f^{-}(y) d y .
$$

Monge's problem is to find among one-to-one mappings $s: \Lambda \rightarrow \Lambda$ which transfer $\mu^{+}$onto $\mu^{-}$the one that minimizes the work functional

$$
I[s]=\int_{\Lambda} c(x, s(x)) \mu^{+}(d x),
$$

where $c(x, y)$ is a given continuous nonnegative function on $\Lambda \times \Lambda$. The function $c(x, y)$ can be interpreted as the cost of transportation of a unit mass from $x$ to $y$. Two important examples of $c(x, y)$ in the case $\Lambda=\mathbf{R}^{n}$ are the following:

$$
\begin{array}{cl}
\text { cost }=\text { distance }: & c(x, y)=|x-y|, \\
\text { cost }=\text { distance }^{2}: & c(x, y)=|x-y|^{2} .
\end{array}
$$

A number of works study these two cases, see [E] and references therein.

In the rest of the paper will consider the case

$$
\text { cost }=\text { distance in } \Lambda: \quad c(x, y)=d_{\Lambda}(x, y) .
$$

An important step in the study of Monge's problem is a dual problem introduced by Kantorovich. In the case (15) the dual problem is the following. Find a potential $u \in$ $\operatorname{Lip}_{1}(\Lambda)$ maximizing

$$
K[u]=\int_{\Lambda} u\left(f^{+}-f^{-}\right) d x
$$

among the functions that belong to $\operatorname{Lip}_{1}(\Lambda)$.

Following the calculation of Remark 3 , we see that $u$ maximizes (16) among the $\operatorname{Lip}_{1}(\Lambda)$ functions if and only if

$$
f^{+}-f^{-} \in \partial I_{\Lambda}^{\infty}[u]
$$

In the case when $\Lambda=\mathbf{R}^{n}$ (and then (15) becomes $c(x, y)=|x-y|$ ) a more precise form of (17) was discovered in [EGan]. Let $f^{+}, f^{-}$be bounded and Lipschitz. Then there exists a function $a \in L^{\infty}\left(\mathbf{R}^{n}\right)$ such that

$$
a \geq 0, \quad \operatorname{supp} a \subset\{|D u|=1\},
$$


and

$$
-\operatorname{div}(a D u)=f^{+}-f^{-} \quad \text { in } \mathbf{R}^{n}
$$

in the weak sense, where $D u=\left(\partial_{x_{1}} u, \ldots, \partial_{x_{n}} u\right)$ is the gradient. The geometry of mass transport is then described in the terms of $a, D u$. Namely, under technical assumptions on $f^{+}, f^{-}$, the optimal map $s: \mathbf{R}^{n} \rightarrow \mathbf{R}^{n}$ is constructed in [EGan] by solving on each "transport ray" an ODE involving $a, f^{+}, f^{-}$. Transport ray is a longest line segment on which $u$ decreases linearly with rate one. It follows that $D u$ is a constant vector on each transport ray, and the direction of the ray is $-D u$. Thus the optimal mass transfer consists of moving mass along the transport rays. For $x \in \mathbf{R}^{n}$ the $a(x)$ is the rate of mass transport through the point $x$.

Note that if the measures $\mu^{+}$and $\mu^{-}$have concentrations on the sets of lower dimension, then it is possible that a one-to-one mapping $s$ which transfers $\mu^{+}$onto $\mu^{-}$does not exist. For example, $s$ does not exist if $\mu^{+}=\delta(x)$ and $\mu^{-}=f^{-}(y) d y$ where $f^{-} \in L^{\infty}(\Lambda)$. However, the mass transport density $a(x)$ may exist even if the map $s$ does not exist, and the structure of $a(x)$ provides a description of optimal transfer of $\mu^{+}$onto $\mu^{-}$. In the next section we will consider such case.

Now we turn to the interpretation of the model (3). Comparing (3) with (17) we see that $u(\cdot, t)$ is a Monge-Kantorovich potential corresponding to the problem of optimal transfer of $\mu^{+}=f(x, t) d x$ onto $\mu^{-}=\partial_{t} u(y, t) d y$ in $\Lambda$ with the cost function $d_{\Lambda}(\cdot, \cdot)$. Thus heuristically the model (3) implies the following. At time $t>0$ sand is added at the rate $f(\cdot, t)$. Sand particles slide downhill along the lines of steepest descent of the sandpile (i.e., in the direction $-D u$ ) in order to keep the slope of sandpile less than or equal to one. As the result of sand sliding the incoming sand density $f(\cdot, t)$ is getting instantaneously reallocated into another density $f^{-}(\cdot, t)$. This new density $f^{-}(\cdot, t)$ becomes the instantaneous rate of increase of the sandpile height at time $t$, i.e. $f^{-}(\cdot, t)=\partial_{t} u(\cdot, t)$. The optimality condition

$$
f(\cdot, t)-\partial_{t} u(\cdot, t) \in \partial I_{\Lambda}^{\infty}[u(\cdot, t)] \quad \text { for a. e. } \quad t>0
$$

implies in particular that sand motion may occur only through the regions of sandpile surface where the slope is equal to one.

In order to understand how sand moves in the presence of an obstacle we construct in the next section the mass transport density in the case of a single point source. This mass transport density is a nonnegative Radon measure that has concentrations on the sets of lower dimension.

\section{Transport rays}

From now on we consider the solution of the problem (3) in the dimension two, with a single point source and zero initial data. Our goal is to define and construct the mass transport density for this solution. We do that in Section 5. In this section we introduce notation and prove some preliminary facts used in Section 5. Specifically, we describe the structure of the spatial gradient of the solution.

Let $\Omega \subset \mathbf{R}^{2}$ be an open convex bounded set with $C^{2}$ boundary. We assume that $\partial \Omega$ is strictly convex in the sense that the curvature $\kappa(x)$ of $\partial \Omega$ at $x$ is strictly positive for every $x \in \partial \Omega$. It follows that

$$
\kappa(x) \geq C>0 \quad \text { for every } \quad x \in \partial \Omega .
$$


Let $\Lambda=\mathbf{R}^{n} \backslash \Omega$. Let $O \in \Lambda^{0}$, where $O=(0,0)$ is the origin in $\mathbf{R}^{2}$. Let

$$
f(x, t)=f_{0}(t) \delta(x), \quad g(x) \equiv 0,
$$

where $\delta(x)$ is the Dirac mass at $O$. Then, according to Lemma 4 and Proposition 5 , the unique solution of $(3)$ is

$$
u(x, t)=\max \left\{0, z(t)-d_{\Lambda}(x, O)\right\}
$$

where $z(t)$ satisfies $(9)$. Let $D(t)$ be the set (8). Now we have $g \equiv 0$ and so

$$
D(t)=\left\{x \in \Lambda \mid d_{\Lambda}(x, O)<z(t)\right\} .
$$

Now we discuss the structure of $D(t)$ and of $D u(\cdot, t)=\left(\partial_{x_{1}} u(\cdot, t), \partial_{x_{2}} u(\cdot, t)\right)$.

In the case $z(t) \leq \operatorname{dist}(O, \Omega)$ the obstacle does not affect the set $D(t)$, and thus $D(t)$ is a disk of radius $z(t)$. Consequently, $u(\cdot, t)$ is differentiable on $D(t) \backslash\{O\}$ and $D u(x, t)=\frac{x-O}{|x-O|}$ for any $x \in D(t) \backslash\{O\}$.

Consider the case

$$
z(t)>\operatorname{dist}(O, \Omega)
$$

If $x, y \in \Lambda^{0}$, then a shortest path in $\Lambda$ connecting $x$ and $y$ (denote it $\mathrm{P}(\mathrm{x}, \mathrm{y})$ ) has the following structure. If the straight line segment $x y$ connecting $x$ and $y$ does not intersect $\Omega$, then

$$
P(x, y)=x y
$$

Otherwise

$$
P(x, y)=x x^{\prime} \cup T \cup y y^{\prime},
$$

where $x x^{\prime}, y y^{\prime}$ are straight line segments and $T$ is an arc of $\partial \Lambda$. Here $x^{\prime}, y^{\prime} \in \partial \Lambda$ are such points that the segments $x x^{\prime}$ and $y y^{\prime}$ are tangent to $\partial \Lambda$ at $x^{\prime}$ and $y^{\prime}$ respectively. $T$ is the shorter arc of $\partial \Lambda$ between $x^{\prime}$ and $y^{\prime}$. Figure 1 shows $P(x, y)$ in both cases.

Note that there can be up to 2 shortest paths in $\Lambda$ connecting $x$ and $y$ (since, by convexity of $\Omega$, there are two tangent to $\Omega$ rays beginning at a point of $\Lambda^{0}$ ). Denote

$$
\mathcal{R}=\{x \in \Lambda \mid \text { there exist } 2 \text { shortest paths connecting } x \text { and } O \text { in } \Lambda\},
$$

see Figure 2 (the paths $x A B O$ and $x C D O$ have the same length). For $x \in \Lambda \backslash \mathcal{R}$ denote $P(x)$ the unique shortest path connecting $x$ and $O$. If $x \in D(t) \backslash \mathcal{R}$ then $P(x) \subset D(t) \backslash \mathcal{R}$.

Since the obstacle $\Omega$ is a convex set with smooth boundary, a standard calculation with the use of Implicit Function Theorem shows that $\mathcal{R}$ is a smooth curve without selfintersections. In addition, $\mathcal{R}$ intersects $\partial \Lambda$ at one point.

Denote

$$
\partial^{\text {int }} D(t)=\{O\} \cup \mathcal{R} \cup(\partial D(t) \backslash \partial \Lambda) .
$$

We call $\partial^{\text {int }} D(t)$ the interior boundary of $D(t)$. Let $x \in \Lambda \backslash \partial^{\text {int }} D(t)$. Then there exists a unique shortest path in $\Lambda$ connecting $x$ and $O$ (denote this path $P(x)$ ). Same is true for every point in some neighborhood of $x$. Then using (22), smoothness and strict convexity of $\partial \Omega$, and taking into account the structure of a shortest path in $\Lambda$ discussed above, we conclude that

$$
D u(\cdot, t) \in C\left(\Lambda \backslash \partial^{\mathrm{int}} D(t) ; \mathbf{R}^{2}\right) .
$$




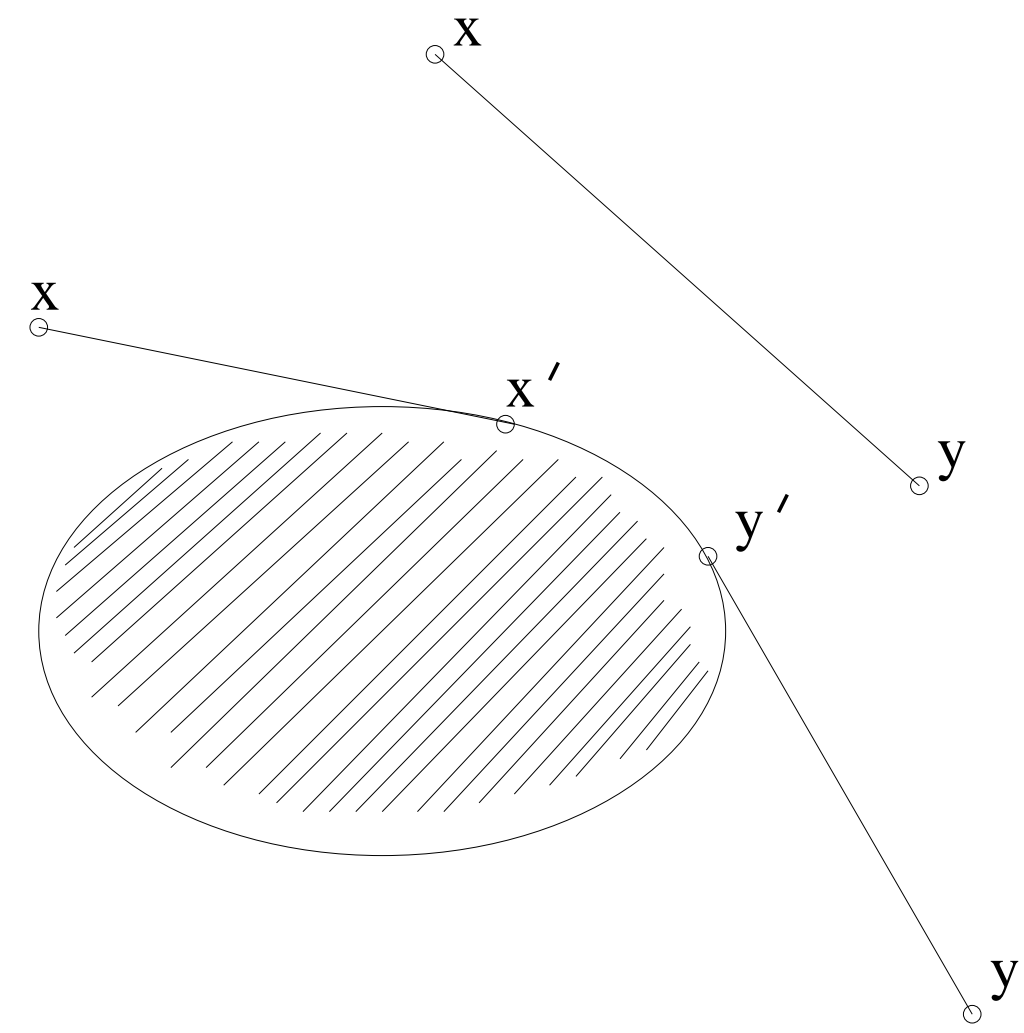

Figure 1: Shortest paths in $\Lambda$

We clearly have:

$$
|D u(\cdot, t)| \equiv 1 \quad \text { on } D(t) \backslash \partial^{\text {int }} D(t), \quad \text { and }\|D u\|_{L^{\infty}(\Lambda)} \leq 1 .
$$

The more detail structure of $D u$ is following. By $(22), u(\cdot, t)$ increases linearly with rate one on each line segment which is a part of a shortest path in $\Lambda$ connecting $x \in D(t)$ with $O$. But $|D u| \leq 1$. Thus in the relative interior of each such segment $D u$ is a constant unit vector collinear to the segment.

Let $x \in D(t) \backslash \partial^{\text {int }} D(t)$. Denote

$$
R_{x}=\{y \in \Lambda|| u(x)-u(y)|=| x-y \mid\} .
$$

It follows from (27), (28) that $R_{x}$ is a line segment. We call $R_{x}$ a transport ray through $x$. It follows from (22) that

$$
\left(R_{x}\right)^{0} \subset D(t) \backslash \partial^{\text {int }} D(t),
$$

where $\left(R_{x}\right)^{0}$ is the relative interior of $R_{x}$. Thus by (27)

$$
u(\cdot, t) \quad \text { is differentiable in }\left(R_{x}\right)^{0} \text { for any } x \in D(t) .
$$

For $x \in D(t) \backslash \partial \Lambda$ any shortest path in $\Lambda$ between $x$ and $O$ contains a line segment. Denote this segment $\tilde{R}_{x}$. One endpoint of $\tilde{R}_{x}$ is $x$. From (22), $\tilde{R}_{x} \subset R_{x}$. Thus for every $x \in D(t) \backslash \partial^{\text {int }} D(t)$ there exists a transport ray $R_{x}$ of positive length through $x$, and $D u$ in $\left(R_{x}\right)^{0}$ is a constant unit vector collinear to $R_{x}$. It also follows that each transport ray 


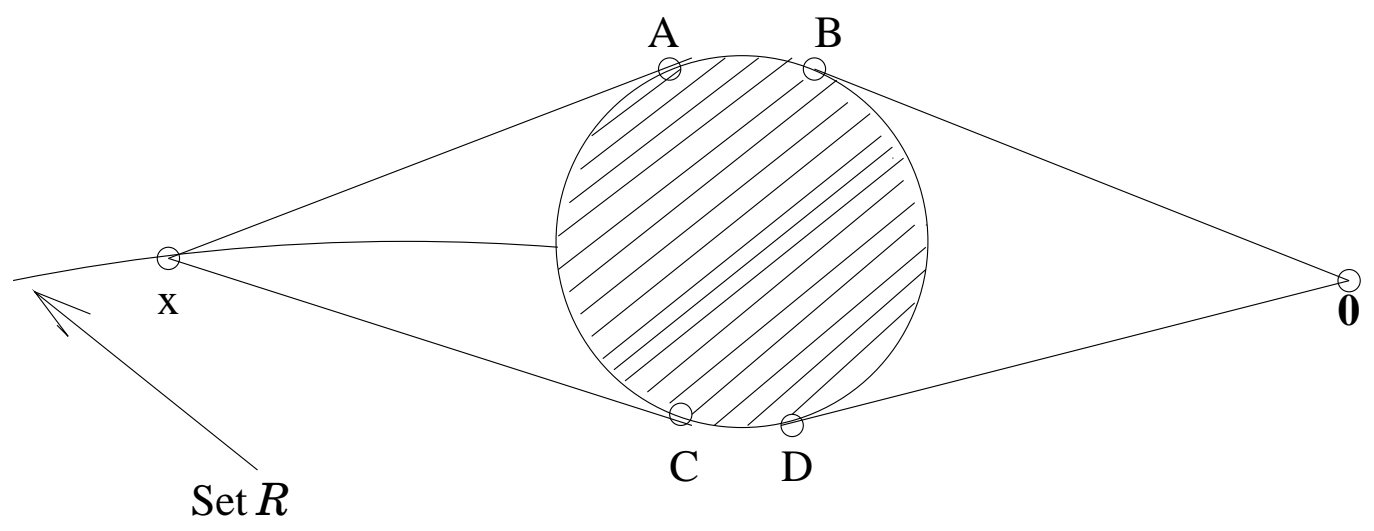

Figure 2: Set $\mathcal{R}$.

is the longest segment in $D(t)$ on which the function $x \rightarrow d_{\Lambda}(x, O)$ increases linearly with rate one.

If $R_{1}$ and $R_{2}$ are two transport rays, then $\left(R_{1}\right)^{0} \cap\left(R_{2}\right)^{0}=\emptyset$. This follows from (29) and (30).

Let $a$ and $b$ be the endpoints of a transport ray $R$ such that $u(a)>u(b)$. We call $a$ the upper end of $R, b$ the lower end of $R$. We denote $\hat{u}(R)$ the upper end of $R$, and $\hat{l}(R)$ the lower end of $R$. Segments $O M, O L, O E, C D, F G$ on Figure 3 are examples of transport rays in the case when the set $D(t) \cap \mathcal{R}$ is empty. Segments $O M, O L, C D, F G$ on Figure 4 are examples of transport rays in the case when the set $D(t) \cap \mathcal{R}$ is nonempty.

The following proposition describes the basic properties of distance rays.

Proposition 6 (a) For any distance ray $R$

$$
\begin{array}{llll}
\text { either } & \hat{u}(R)=O & \text { and } & \hat{l}(R) \in \partial D(t), \\
\text { or } & \hat{u}(R) \in \partial \Lambda & \text { and } & \hat{l}(R) \in \partial^{i n t} D(t) .
\end{array}
$$

(b) If $\hat{u}(R) \in \partial \Lambda$ then $R$ is tangent to $\Lambda$ at $\hat{u}(R)$.

(c) $x \in \partial \Lambda$ cannot be the upper end of one transport ray $R_{1}$ and the lower end of another transport ray $R_{2}$.

Proof. From the structure of a shortest path between $x \in D(t)$ and $O$ we conclude that for any transport ray $R_{x}$

$$
\text { either } \hat{u}\left(R_{x}\right)=O \text {, or } \hat{u}\left(R_{x}\right) \in \partial \Lambda \text { and } R_{x} \text { is tangent to } \partial \Lambda \text { at } a=\hat{u}\left(R_{x}\right) \text {. }
$$

Thus (b) follows.

The lower end of any transport ray $R_{x}$ satisfies

$$
\hat{l}\left(R_{x}\right) \in \partial D(t) \cup \mathcal{R} .
$$

Indeed, suppose $b=\hat{l}\left(R_{x}\right) \in D(t) \backslash(\partial D(t) \cup \mathcal{R})$. Then we can extend $R_{x}$ beyond $b$ until it intersects $\partial D(t) \cup \mathcal{R}$ at a point $c$. Denote the extended segment $R^{\prime}$. Clearly, $R^{\prime}$ contains $R_{x}$. Also, $R^{\prime}$ is a part of a shortest path in $\Lambda$ between $c$ and $O$ since $R_{x}$ is a part of the shortest path from $b$ to $O$ and $\left(R^{\prime}\right)^{0}$ does not intersect $\mathcal{R}$. Thus $u(x, t)-u(c, t)=|x-c|$, i.e., $c \in R_{x}$ - a contradiction. 


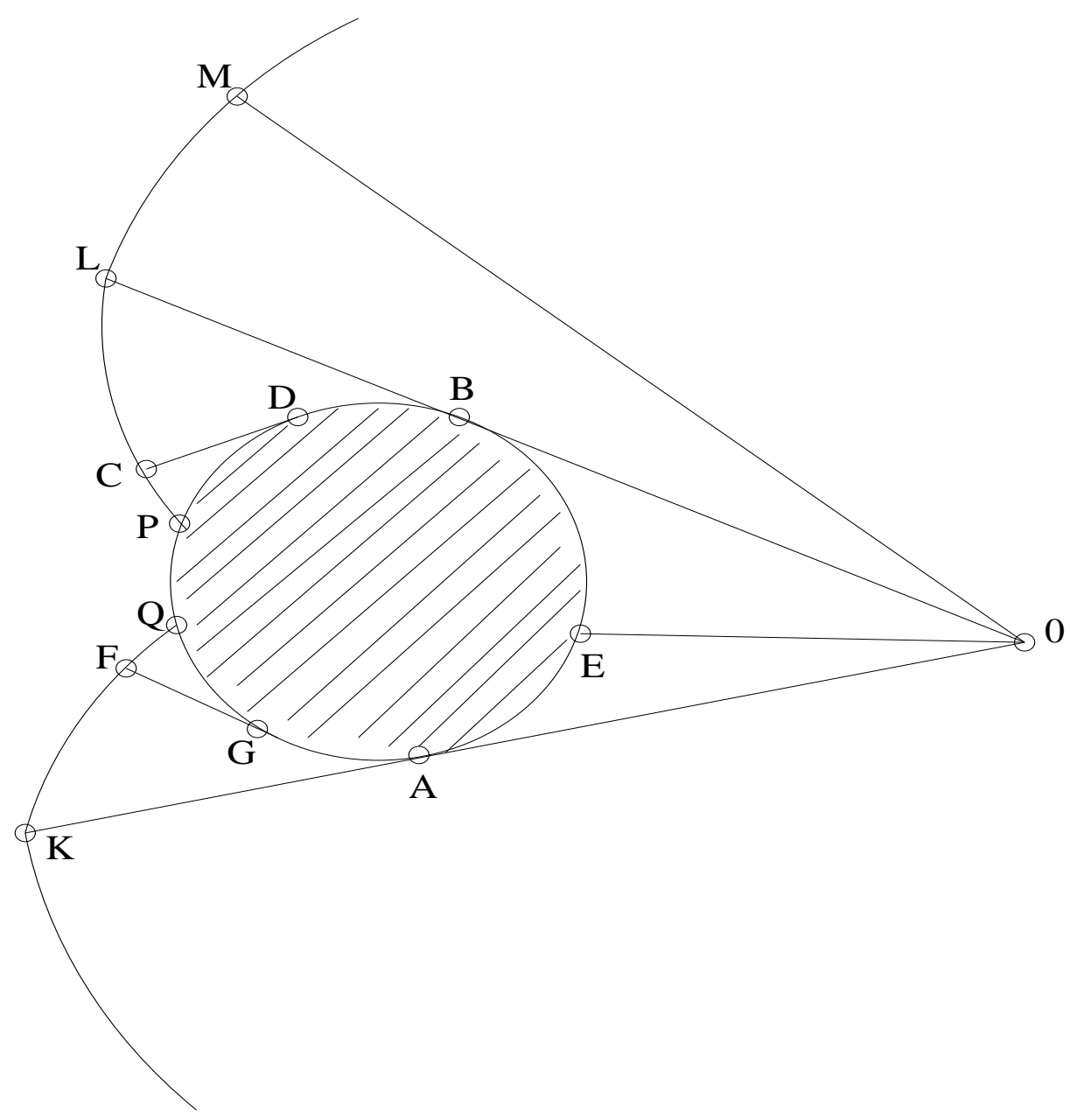

Figure 3: Structure of $D(t)$. Case 1: $D(t) \cap \mathcal{R}=\emptyset$

If $\hat{l}\left(R_{x}\right) \in \mathcal{R}$ then $\hat{u}\left(R_{x}\right) \in \partial \Lambda$ (proof: there are two shortest paths between $\hat{l}\left(R_{x}\right)$ and $O$ in $\Lambda$, thus each of these paths cannot be a segment of a straight line from $\hat{l}\left(R_{x}\right)$ to $O$. Thus each of these paths has the form (25). The desired conclusion follows).

The above discussion confirms (a).

Proof of (c). Suppose $x=\hat{u}\left(R_{1}\right)=\hat{l}\left(R_{2}\right)$. If the rays $R_{1}$ and $R_{2}$ are collinear then $R=R_{1} \cup R_{2}$ is a transport ray and $x$ lies in the relative interior of $R$ - a contradiction. Suppose now that $R_{1}$ and $R_{2}$ are not collinear. Since $\hat{u}\left(R_{1}\right)=\hat{l}\left(R_{2}\right) \in \partial \Lambda$ then it follows from (32) that $\hat{u}\left(R_{2}\right)=O, \hat{l}\left(R_{1}\right) \in \partial D(t) \backslash \partial \Lambda$. Thus the shortest path in $\Lambda$ between $\hat{l}\left(R_{1}\right)$ and $O$ is the union of two line segments $R_{1}$ and $R_{2}$ which are not collinear. But a shortest path in $\Lambda$ between two points can be either like (24) or like (25) - a contradiction.

Proposition 6 is proved.

The following examples illustrate Proposition 6 (a). The ray satisfying $\hat{u}(R) \in \partial \Lambda$, $\hat{l}(R) \in \mathcal{R}$ is $F G$ on Figure 4. The rays satisfying $\hat{u}\left(R_{x}\right) \in \partial \Lambda, \hat{l}\left(R_{x}\right) \in \partial D(t)$ are $C D$ on Figure 3 and $C D$ on Figure 4. Examples of the case $\hat{u}\left(R_{x}\right)=O$ are the rays $M O, E O$ and $L O$ on Figure 3. 


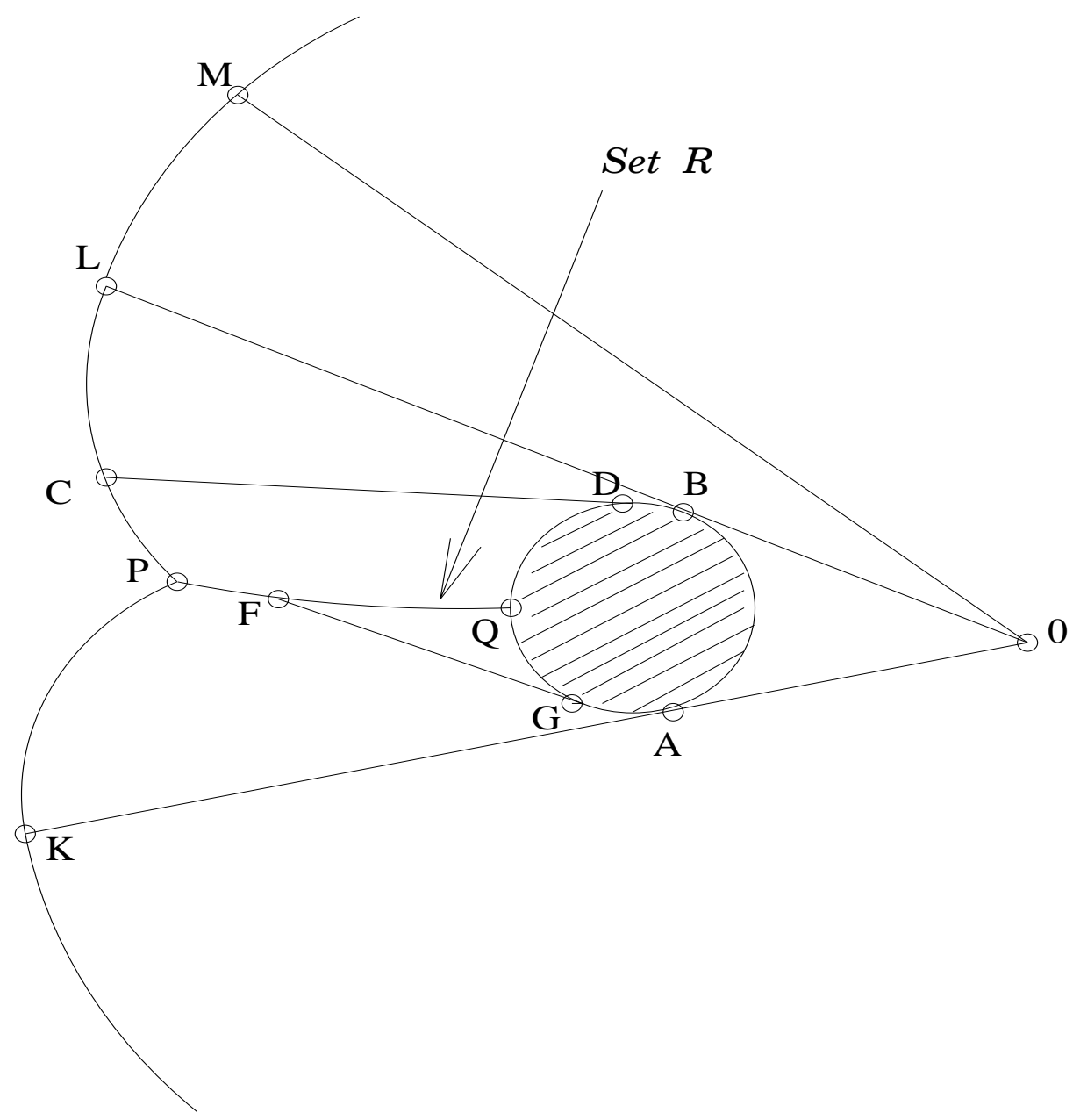

Figure 4: Structure of $D(t)$. Case 2: $D(t) \cap \mathcal{R} \neq \emptyset$

Denote

$$
\partial^{\tan } D(t)=\{a \in \partial \Lambda \mid a=\hat{u}(R) \text { for some transport ray } R\} .
$$

We have showed above that if $R$ is a transport ray and $\hat{u}(R) \in \partial \Lambda$, then $R$ is tangent to $\partial \Lambda$ at $\hat{u}(R)$. Thus we call $\partial^{\tan } D(t)$ the tangential boundary of $D(t)$. Let $A, B \in \partial \Lambda$ be the contact points of $\partial \Lambda=\partial \Omega$ with the rays tangent to $\Omega$ and starting at $O$. Denote $R_{\text {tan }}^{1}$ the segment $O A$, and $R_{\mathrm{tan}}^{2}$ the segment $O B$. We can assume that $\left|R_{\mathrm{tan}}^{1}\right| \leq\left|R_{\mathrm{tan}}^{2}\right|$. The set $\partial^{\tan } D(t)$ is empty if $z(t) \leq\left|R_{\tan }^{1}\right|=\min \left(\left|R_{\tan }^{1}\right|,\left|R_{\tan }^{2}\right|\right)$ and is nonempty otherwise.

Let $x \in \partial^{\tan } D(t)$. Then there exists a unique shortest path $P(x)$ in $\Lambda$ connecting $x$ and $O$. The point $x$ is the upper end of a transport ray. Thus $P(x)$ is not a line segment connecting $x$ and $O$. It follows that $P(x)$ is the union of an $\operatorname{arc} T \subset \partial \Omega$ and a line segment tangent to $\Omega$ with one endpoint at $O$. Thus this line segment is either $R_{\text {tan }}^{1}$ or $R_{\text {tan }}^{2}$. In addition, $\partial^{\tan } D(t) \cap \mathcal{R}=\emptyset$ by Proposition 6 (a). Now it is easy to see that the set $\partial^{\tan } D(t)$ has the following structure.

If $\left|R_{\mathrm{tan}}^{2}\right| \geq z(t)>\left|R_{\mathrm{tan}}^{1}\right|$ then $\partial^{\tan } D(t)$ is a connected open arc, denote it $T_{1}$. One endpoint of $T_{1}$ is $A$.

If $z(t)>\left|R_{\tan }^{2}\right|=\max \left(\left|R_{\tan }^{1}\right|,\left|R_{\tan }^{2}\right|\right)$ then $\partial^{\tan } D(t)$ is the union of two disjoint open 
arcs, denote them $T_{1}$ and $T_{2}$. One endpoint of $T_{1}$ is $A$. One endpoint of $T_{2}$ is $B$. If $D(t) \cap \mathcal{R} \neq \emptyset$ (and then $D(t) \cap \mathcal{R}$ consists of one point, denote it $Q$ ) then the arcs $T_{1}$ and $T_{2}$ have the common second endpoint, the point $Q$.

On Figure 3 the $T_{1}$ and $T_{2}$ are the open $\operatorname{arcs} A Q$ and $B P$. On Figure 4 the $T_{1}$ and $T_{2}$ are the open arcs $A Q$ and $B Q$.

Below, in order to consider all cases at once, we will refer to both $T_{1}$ and $T_{2}$, but depending on $z(t)$ one of or both $T_{1}$ and $T_{2}$ can be empty as we discussed.

Define the following sets

$$
\begin{aligned}
D^{k} & =\left\{x \in D(t) \backslash\left(\partial^{\text {int }} D(t) \cup \partial \Lambda\right) \mid \hat{u}\left(R_{x}\right) \in T_{k}\right\}, \text { where } k=1,2 ; \\
D^{3} & =\left\{x \in D(t) \backslash\left(\partial^{\text {int }} D(t) \cup \partial \Lambda\right) \mid \hat{u}\left(R_{x}\right)=O\right\} .
\end{aligned}
$$

The sets $D^{1}, D^{2}, D^{3}$ are disjoint by Proposition 6 (c), and

$$
D(t) \backslash\left(\partial^{\text {int }} D(t) \cup \partial \Lambda\right)=D^{1} \cup D^{2} \cup D^{3}
$$

since the upper end of every transport ray is either the point $O$ or belongs to $T_{1} \cup T_{2}$.

On Figure 3 the sets $D^{1}$ and $D^{2}$ are the open regions $A K Q$ and $B P L$. On Figure 4 the sets $D^{1}$ and $D^{2}$ are the open regions $A K P Q$ and $B L P Q$.

For $x \in D(t)$ define the set

$$
S(x)=\left\{y \in D(t) \backslash\left(\partial^{\text {int }} D(t) \cup \partial \Lambda\right) \mid x \in P(y)\right\} .
$$

We have

$$
D^{1}=S(A), \quad D^{2}=S(B)
$$

We also have

$$
S(x)=D^{k} \cup\left\{y \in R_{\tan }^{k} \mid d_{\Lambda}(O, y)>d_{\Lambda}(O, x)\right\} \quad \text { for } x \in R_{\tan }^{k}, k=1,2 .
$$

Finally, we have

$$
S(x)=\cup_{y \in T(x)}\left(R_{y}\right)^{0} \quad \text { for } x \in T_{k}, \quad k=1,2,
$$

where

$$
T(x)=\left\{y \in T_{k} \mid d_{\Lambda}(O, y)>d_{\Lambda}(O, x)\right\} \quad \text { for } x \in T_{k}, \quad k=1,2 .
$$

On Figure 3 the sets $T_{1}$ and $T_{2}$ are the open $\operatorname{arcs} A Q$ and $B P$. The point $G$ lies on $T_{1}$. The set $S(G)$ is the open region $G F Q$. The set $T(G)$ is the open arc $G Q$.

On Figure 4 the $T_{1}$ and $T_{2}$ are the open arcs $A Q$ and $B Q$. The point $G$ lies on $T_{1}$. The set $S(G)$ is the open region $G F Q$, the set $T(G)$ is the open arc $G Q$. The point $D$ lies on $T_{2}$. The set $S(D)$ is the open region $D C P Q$, the set $T(D)$ is the open arc $D Q$.

\section{$5 \quad$ Geometry of mass transfer}

We continue to consider the solution (22) of the problem (3) in the dimension two. We use assumptions about the obstacle $\Omega$ and notation introduced in Section 4 .

First we will define mass transport density $a$. The motivation of Definition 7 below is following. In our case $a$ is a Radon measure. The measure $a$ should satisfy equation (19) in a weak sense. According to the Monge-Kantorovich interpretation of the model (3), the equation (19) in our case has the form

$$
-\operatorname{div}(a D u)=f-\partial_{t} u \quad \text { for a. e. } \quad t>0 .
$$


In order to have the left-hand side of (39) properly defined, we need to impose suitable conditions on the measure $a$, based on the properties (28) and (27) of Du.

Definition 7 Let $f, g$ be defined by (21), and let $u$ be the solution of (3) (and then $u$ is defined by (22)). Let $t>0$. A nonnegative Radon measure a on $\Lambda$ is called a mass transport density at time $t$ if

(a) $\lim _{r \rightarrow+0} a\left(B_{r}(O)\right)=0$;

(b) For any $R>0$

$$
\begin{aligned}
& \lim _{\varepsilon \rightarrow+0} a\left(\left\{x \in \Lambda \mid d_{\Lambda}(x, O) \in(R-\varepsilon, R+\varepsilon)\right\}\right)=0, \\
& \lim _{\varepsilon \rightarrow+0} a\left(\left\{x \in \Lambda \mid d_{\Lambda}(x, O) \leq R, \quad \operatorname{dist}(x, \mathcal{R}) \leq \varepsilon\right\}\right)=0 ;
\end{aligned}
$$

(c) $\operatorname{supp}(a) \subset \overline{D(t)}$;

$$
\int_{\Lambda}\left(f(x, t)-\partial_{t} u(x, t)\right) \varphi(x) d x=\int_{\Lambda} D u(x, t) D \varphi(x) d a(x) .
$$

Remark 8 The conditions (a) and (b) record the requirement that the measure a does not have concentrations at $O$ and on the sets $\mathcal{R}$ and $\left\{d_{\Lambda}(x, O)=R\right\}$ for any $R>0$.

Remark 9 For the functions $f, g$ and $u$ defined by (21), (22) the equality (42) reduces to

$$
\frac{f_{0}(t)}{|D(t)|} \int_{D(t)}(\varphi(O)-\varphi(x)) d x=\int_{D(t)} D u(x, t) D \varphi(x) d a(x) .
$$

The conditions (a) and (b) of Definition 7 and (27) imply that the right-hand side of (43) is well defined.

First we prove a stronger version of the property (41) of mass transport density.

Lemma 10 Let a be a mass transport density at time $t \geq 0$. Then for each $t$

$$
\lim _{\varepsilon \rightarrow+0} \frac{1}{\varepsilon} a(\{x \in D(t) \mid \operatorname{dist}(x, \mathcal{R}) \leq \varepsilon\})=0 ;
$$

Proof. Fix $t>0$. If $D(t) \cap \mathcal{R}=\emptyset$, then nothing to prove. Thus we assume that

$$
D(t) \cap \mathcal{R} \neq \emptyset .
$$

In this case the intersection of the sets $\partial D(t) \backslash \partial \Lambda$ and $\mathcal{R}$ consists of one point, we denote by $P$ this point (see Figure 4 ).

We use the following properties of transport rays. The curve $\mathcal{R}$ is orthogonal to $\partial \Lambda$ at the point of their intersection. In addition, let $x \in \mathcal{R}$, and let the two transport rays $R_{x, 1} \subset D^{1}$ and $R_{x, 2} \subset D^{2}$ be such that $x=\hat{l}\left(R_{x, 1}\right)=\hat{l}\left(R_{x, 2}\right)$ (such rays exist by definition of $\mathcal{R})$. Denote $\tau_{\mathcal{R}}(x)$ the unit vector tangent to $\mathcal{R}$ at $x$ and oriented in the direction towards $\Omega$. Denote $\alpha\left(e_{1}, e_{2}\right)$ the angle between the vectors $e_{1}, e_{2}$. We have: the functions $x \rightarrow$ $\alpha\left(R_{x, 1}, \tau_{\mathcal{R}}(x)\right)$ and $x \rightarrow \alpha\left(R_{x, 2}, \tau_{\mathcal{R}}(x)\right)$ are continuous on $\mathcal{R}$, and $\frac{\pi}{2} \geq \alpha\left(R_{x, k}, \tau_{\mathcal{R}}(x)\right)>0$ for any $x \in \mathcal{R}, \mathrm{k}=1,2$. Here the direction of transport rays chosen from the lower to the 
upper end. The above facts follow from smoothness and strict convexity of $\partial \Omega$. In particular it follows that for each $t$ there exists $\alpha_{0}>0$ such that

$$
\frac{\pi}{2} \geq \alpha\left(R_{x, k}, \tau_{\mathcal{R}}(x)\right) \geq \alpha_{0}>0 \quad \text { for any } x \in \mathcal{R} \cap D(t), \quad k=1,2 .
$$

Now we prove the Lemma. Let $\eta \in C^{\infty}\left(\mathbf{R}^{1}\right)$ satisfy

$$
0 \leq \eta \leq 1 \quad \text { on } \mathbf{R}^{1}, \quad \eta(s) \equiv 1 \quad \text { for }|s|<1, \quad \eta(s) \equiv 0 \text { for }|s|>2 .
$$

Let $\varepsilon>0$. Define function $\eta_{\varepsilon}(s)$ by

$$
\eta_{\varepsilon}(s)=\frac{1}{\varepsilon} \eta\left(\frac{1}{\varepsilon} s\right)
$$

Let $\mathcal{P}$ be the union of interiors of all segments beginning at points of $\mathcal{R}$ and tangent to $\partial \Omega$, i.e.,

$$
\begin{gathered}
\mathcal{P}=\left\{x \in \Lambda^{0} \backslash \mathcal{R} \quad \mid \quad x \text { lies on a shortest path } P^{x}(y) \text { between some } y \in \mathcal{R} \text { and } O,\right. \\
\text { and segment } x y \text { lies in } \Lambda\} .
\end{gathered}
$$

From strict convexity of $\Omega$ we see that $\mathcal{P}$ is an open set and

for every $R>0$ there exists $\varepsilon>0$ such that $\left[N_{\varepsilon}(\mathcal{R}) \backslash \mathcal{R}\right] \cap B_{R}(O) \subset \mathcal{P} \cap B_{R}(O)$.

Here $N_{\varepsilon}(\cdot)$ denotes $\varepsilon$ neighborhood in $\mathbf{R}^{2}$ of a set. If $x \in \mathcal{P}$ then the point $y \in \mathcal{R}$ and the path $P^{x}(y)$ from the above definition are defined uniquely for $x$. Thus we can define a map $\hat{r}: \mathcal{P} \rightarrow \mathcal{R}$ by $\hat{r}(x)=y$. Since $\mathcal{R}$ is a $C^{1}$ curve and $\partial \Omega$ is convex and smooth, it follows from (46) that $r \in C^{1}\left(\mathcal{P}, \mathbf{R}^{2}\right)$.

Define $\psi_{\varepsilon}: \Lambda \rightarrow \mathbf{R}^{1}$ by

$$
\psi_{\varepsilon}(x)= \begin{cases}\eta_{\varepsilon}(0) & \text { if } x \in \mathcal{R} \\ \eta_{\varepsilon}\left(d_{\Lambda}(x, \hat{r}(x))\right. & \text { if } x \in \mathcal{P} \\ 0 & \text { otherwise }\end{cases}
$$

Using (49) and properties of $\eta_{\varepsilon}$ and $\hat{r}$ we see that $\psi_{\varepsilon} \in C^{1}(\Lambda)$ if $\varepsilon$ is small. Define $\varphi_{\varepsilon}: \Lambda \rightarrow$ $\mathbf{R}^{1}$ as following. Let

$$
\varphi_{\varepsilon}(x)=\int_{P(x)} \psi_{\varepsilon} d \mathcal{H}^{1} \quad \text { for } \quad x \in \Lambda \backslash \mathcal{R},
$$

where $P(x)$ is the unique shortest path in $\Lambda$ between $x \in \Lambda \backslash \mathcal{R}$ and $O$. Since $P(x)$ is a $C^{1}$ curve, we have

$$
\begin{array}{llrl}
\varphi_{\varepsilon}(x) & =\int_{d_{\Lambda}(\hat{r}(x), x)}^{d_{\Lambda}(\hat{r}(x), O)} \eta_{\varepsilon}(s) d s & & \text { if } x \in \mathcal{P}, \\
\varphi_{\varepsilon}(x) & =0 & \text { if } x \in \Lambda \backslash \overline{\mathcal{P}} .
\end{array}
$$

Now we define $\varphi_{\varepsilon}$ by (51) on $\overline{\mathcal{P}}$, and by (52) on $\Lambda \backslash \overline{\mathcal{P}}$. Thus $\varphi_{\varepsilon}$ is defined on $\Lambda$ and (50) holds.

We have $d_{\Lambda}(\cdot, O) \in C^{1}(\Lambda \backslash(\mathcal{R} \cup\{O\}))$. Then (49) and the properties of $\eta_{\varepsilon}(\cdot)$ and $\hat{r}(\cdot)$ imply that for $\varepsilon>0$ small enough

$$
\varphi_{\varepsilon} \in C^{1}\left(\Lambda \cap B_{2 R}(O)\right. \text {.) }
$$


Now from (50)

$$
D \varphi_{\varepsilon}(x) D u(x)=-\psi_{\varepsilon}(x) \text { on } \Lambda \cap B_{2 R}(O), \quad \text { and } \quad \varphi_{\varepsilon}(O)=0 .
$$

Inserting $\varphi_{\varepsilon}$ into (43) and using (53) we get

$$
\int_{\Lambda} \psi_{\varepsilon} d a=\frac{f_{0}(t)}{|D(t)|} \int_{D(t)} \varphi_{\varepsilon} d x
$$

From (47), (48), (50)

$$
\left|\varphi_{\varepsilon}\right| \leq C \quad \text { on } \quad \overline{D(t)}, \quad \operatorname{supp} \varphi_{\varepsilon} \subset N_{\varepsilon}(\mathcal{R}),
$$

where $C$ does not depend on $\varepsilon$.

Since $\mathcal{R}$ is a $C^{1}$ curve, we get

$$
\left|N_{\varepsilon}(\mathcal{R}) \cap D(t)\right|=O(\varepsilon) .
$$

Denote

$$
N_{\Lambda, \varepsilon}(\mathcal{R})=\left\{x \in \mathcal{P} \quad \mid \quad d_{\Lambda}(x, \hat{r}(x))<\varepsilon\right\} .
$$

By (47), (48)

$$
\psi_{\varepsilon} \equiv \frac{1}{\varepsilon} \quad \text { on } \quad N_{\Lambda, \varepsilon}(\mathcal{R}) .
$$

Now from (57) and (54), using (55), (56) we get

$$
0 \leq \frac{1}{\varepsilon} a\left(N_{\Lambda, \varepsilon}(\mathcal{R})\right) \leq \int_{\Lambda} \psi_{\varepsilon} d a=\frac{f_{0}(t)}{|D(t)|} \int_{D(t)} \varphi_{\varepsilon} d x=O(\varepsilon) .
$$

By (46) we have $N_{\frac{\varepsilon}{C}}(\mathcal{R}) \cap D(t) \subset N_{\Lambda, \varepsilon}(\mathcal{R}) \cap D(t)$ where $C$ does not depend on $\varepsilon$. Thus (58) implies (44).

Now we construct the mass transport density $a$. The idea is to rewrite formally the PDE (39) as an ODE with a suitable boundary condition on each transport ray and define the "regular" part of the measure $a$ as the solution of this ODE on each transport ray. We describe this ODE in Remark 13 below. We also need to add the singular part of the measure $a$ which completes the mass balance equation (42).

Denote

$$
D_{\text {sing }}=R_{\text {tan }}^{1} \cup T_{1} \cup R_{\text {tan }}^{2} \cup T_{2} .
$$

We show below that the singular part of the measure $a$ is concentrated on $D_{\text {sing }}$. Note that $D_{\text {sing }}$ is the union of two $C^{1}$ curves $R_{\text {tan }}^{1} \cup T_{1}$ and $R_{\text {tan }}^{2} \cup T_{2}$. Recall that $T_{1}$ and $T_{2}$ are open arcs and thus $\left(R_{\text {tan }}^{1} \cup T_{1}\right) \cap\left(R_{\text {tan }}^{2} \cup T_{2}\right)=\{O\}$. On Figure 3 the set $D_{\text {sing }}$ is $O A \cup A Q \cup O B \cup B P$ (note that this set does not include the points $P$ and $Q$ ). On Figure 4 the set $D_{\text {sing }}$ is $O A \cup A Q \cup O B \cup B Q$ (note that this set does not include the point $Q$ ).

Theorem 11 Let $f, g, u$ be like in the Definition 9. Then for any $t \geq 0$ there exists a unique mass transport density a (in the sense of Definition 7). The measure a has the structure

$$
a=a_{a c} d x+a_{s}
$$


where a ac denotes the absolutely continuous part of a with respect to the 2-dimensional Lebesgue measure, and $a_{s}$ the singular part. The $a_{a c}$ and $a_{s}$ are the following:

$$
a_{a c}(x)= \begin{cases}\frac{f_{0}(t)}{2|D(t)| \rho}\left(r^{2}-\rho^{2}\right), & \text { if } x \in D(t) \backslash \mathcal{R} ; \\ 0, & \text { if } x \in \Lambda \backslash D(t) ;\end{cases}
$$

where

$$
r(x)=\left|R_{x}\right|, \quad \rho(x)=\left|x-\hat{u}\left(R_{x}\right)\right| \quad \text { for } \quad x \in D(t) \backslash \mathcal{R} ;
$$

and

$$
a_{s}=m d \mathcal{H}^{1} \quad \text { on } \quad D_{\text {sing }}, \quad \text { where } \quad m(x)=f_{0}(t) \frac{|S(x)|}{|D(t)|} .
$$

Here $\mathcal{H}^{1}$ is the 1-dimensional Hausdorff measure on $D_{\text {sing }}$.

Remark $12 R_{x}$ is uniquely defined for $x \in D(t) \backslash \mathcal{R}$. This justifies the definitions (61), (60). In addition we have $\mathcal{L}^{2}(\mathcal{R})=0$ since $\mathcal{R}$ is a $C^{1}$ curve. Thus a ac is defined by (60) a. $e$. in $\Lambda$. The measure a defined by (59)-(62) clearly satisfies the properties (a)-(c) of Definition $\%$.

Remark 13 The equation (39) can be formally written as

$$
-D a D u-a \Delta u=f-\partial_{t} u .
$$

We rewrite this as an ODE in the relative interior of any transport ray $R$. Introduce on $R$ the coordinate $\rho$ by $\rho=\rho(x)$ for $x \in R$, where $\rho(x)$ is defined by (61). Then $\rho \in[0, r]$ where $r=|R|$. Since $D u(x, t)=\frac{\hat{u}\left(R_{x}\right)-\hat{l}\left(R_{x}\right)}{\left|\hat{u}\left(R_{x}\right)-\hat{l}\left(R_{x}\right)\right|}$ for $x \in D(t) \backslash \partial^{\text {int }} D(t)$ we have

$$
\frac{d a}{d \rho}=-D a D u \quad \text { on } \quad R .
$$

We will show below (see Remark 15) that

$$
\Delta u(x, t)=-\frac{1}{\rho(x)} \quad \text { for } \quad x \in D(t) \backslash \partial^{i n t} D(t) .
$$

In addition we have by (22), (9)

$$
\partial_{t} u(x, t)=\frac{f_{0}(t)}{|D(t)|} \quad \text { on } \quad D(t) \backslash \partial^{i n t} D(t),
$$

and $f \equiv 0$ in the relative interior of $R$.

Thus we write the equation (63) on $R$ as

$$
\frac{d a}{d \rho}+\frac{1}{\rho} a=-\frac{f_{0}(t)}{|D(t)|}, \quad \rho \in(0, r) .
$$

We add to this equation the boundary condition

$$
a(r)=0,
$$

that records the fact that no mass transfer occurs through the lower end of a ray. The function a ac defined by (60), (61) is the solution of the problem (65), (66) on each transport ray. 
Note that (66) is not an assumption in the model (3). At this point (66) is our guess. But after Theorem 11 is proved, it follows from existence and uniqueness of the mass transport density that (66) is the consequence of (3).

Indeed, by (61) we have $\rho(y)=r(y)$ if $y=\hat{l}(R)$, where $R$ is any transport ray. Thus by (60) $a_{a c}$ is zero at the lower end of any transport ray. By (62) the support of measure $a_{s}$ does not intersect the set of lower ends of transport rays. So there is no mass transfer through lower ends of rays.

Remark 14 Some properties of the measure $a_{s}$ defined by (62) are:

(1) From (36) we have

$$
m \equiv f_{0}(t) \frac{\left|D^{k}\right|}{|D(t)|} \quad \text { on } \quad R_{\text {tan }}^{k}, \quad \text { where } \quad k=1,2 .
$$

(2) If $z(t)<\min \left(\left|R_{\text {tan }}^{1}\right|,\left|R_{\text {tan }}^{2}\right|\right)$ then $m \equiv 0$ since $D^{1}=D^{2}=\emptyset$. Thus in this case $a=a_{a c} d x$.

(3) $m\left(z_{k}\right)=0$ where $\left\{z_{k}\right\}=\partial T_{k} \backslash \partial R_{\text {tan }}^{k}, k=1,2$.

Proof of Theorem 11 We start from the proof of uniqueness. Suppose that the Radon measure $\tilde{a}$ is a mass transport density in the sense of Definition 7 .

As the first step we prove that

$$
\operatorname{supp}\left(\tilde{a}-a_{\mathrm{ac}} d x\right) \subset \bar{D}_{\text {sing }},
$$

where $a_{\text {ac }}$ is defined by (60). First, we calculate the integral

$$
\int_{D(t)} a_{\mathrm{ac}} D u(x, t) D \varphi(x) d x
$$

where $\varphi \in C^{1}\left(\mathbf{R}^{2}\right)$. We use the decomposition (34).

In the domain $D^{3}$ the point $O$ is the upper end of every transport ray, and thus we have $\rho(x)=|x-O|$ where $\rho(x)$ is defined by (61). Then in the polar coordinates $(\rho, \theta)$ with the center $O$, we have $\rho(x)=\rho, r(x)=r(\theta)$ for the functions $\rho(x), r(x)$ defined by (61) and $x \in D^{3}$. Thus by $(60), a_{\text {ac }} \in L^{1}\left(D^{3}\right)$. We clearly have $D u D \varphi=-\frac{\partial \varphi}{\partial \rho}$ in $D^{3}$. Now we calculate using $(60)$

$$
\begin{aligned}
\int_{D^{3}} a_{\mathrm{ac}} D u D \varphi d x & =\frac{f_{0}(t)}{2|D(t)|} \int_{0}^{2 \pi} d \theta \int_{0}^{r(\theta)} \frac{1}{\rho}\left[r^{2}(\theta)-\rho^{2}\right]\left(-\frac{\partial \varphi}{\partial \rho}\right) \rho d \rho \\
& =\frac{f_{0}(t)}{2|D(t)|} \int_{0}^{2 \pi}\left[\varphi(O) r^{2}(\theta)-\int_{0}^{r(\theta)} 2 \rho \varphi d \rho\right] d \theta \\
& =\frac{f_{0}(t)}{|D(t)|} \int_{0}^{2 \pi} \int_{0}^{r(\theta)}[\varphi(O)-\varphi] \rho d \rho d \theta \\
& =\frac{f_{0}(t)}{|D(t)|} \int_{D^{3}}[\varphi(O)-\varphi] d x
\end{aligned}
$$

Now consider the domain $D^{1}$. If $x \in D^{1}$ and $R_{x}$ is the transport ray through $x$, then $\hat{u}\left(R_{x}\right) \in T_{1}$ and $R_{x}$ is tangent to the arc $T_{1}$ at the point $\hat{u}\left(R_{x}\right)$. Define the map $g: D^{1} \rightarrow T_{1}$ and the coordinates $(y, \rho)$ on $D^{1}$ by:

$$
\begin{aligned}
& y=g(x)=\hat{u}\left(R_{x}\right) \in T_{1} \\
& \rho=\left|x-\hat{u}\left(R_{x}\right)\right| .
\end{aligned}
$$


Note that now

$$
\rho(x)=\rho, \quad r(x)=r(y),
$$

where $\rho(x), r(x)$ are the functions $(61)$ and $x \in D^{1}$.

We prove that mapping $g: D^{1} \rightarrow T_{1}$ is locally Lipschitz in $D^{1}$ and compute 1dimensional Jacobian $J_{1} g$ ([Fed69], 3.2.1). Let $x \in D^{1}$ and $y=g(x)$. By (20), in some neighborhood of $y$ the curve $T_{1}$ is a graph $x_{2}=\Psi\left(x_{1}\right)$ in an appropriate Cartesian coordinate system $\left(x_{1}, x_{2}\right)$ on $\mathbf{R}^{2}$, were $\Psi$ a smooth function on $\mathbf{R}^{1}$ satisfying

$$
\Psi^{\prime \prime}>\alpha>0 \text { on } \mathbf{R}^{1} .
$$

Since $D u$ is continuous in $D^{1} \backslash \partial \Lambda$, we have $g\left(B_{\sigma}(x)\right) \subset$ graph $\Psi$ for small $\sigma>0$. Let in coordinates

$$
x=\left(x_{1}, x_{2}\right), \quad y=\left(y_{1}, y_{2}\right)=\left(g_{1}(x), g_{2}(x)\right) .
$$

Then

$$
g_{2}(z)=\Psi\left(g_{1}(z)\right) \text { for } \quad z \in B_{\sigma}(x) .
$$

Since the transport ray $R_{x}$ is tangent to the graph of $\Psi$ at the point $y=g(x)$, we have

$$
x_{2}-\Psi\left(y_{1}\right)=\Psi^{\prime}\left(y_{1}\right)\left(x_{1}-y_{1}\right) .
$$

Changing coordinates if necessary, we can assume that

$$
y_{1}>x_{1} .
$$

Then by (69), (73)

$$
\rho=\left(y_{1}-x_{1}\right) \sqrt{1+\left[\Psi^{\prime}\left(y_{1}\right)\right]^{2}} .
$$

Differentiating (73) we get

$$
\begin{gathered}
\frac{\partial y_{1}}{\partial x_{1}}=\frac{\Psi^{\prime}\left(y_{1}\right)}{\Psi^{\prime \prime}\left(y_{1}\right)\left(y_{1}-x_{1}\right)}, \\
\frac{\partial y_{1}}{\partial x_{2}}=-\frac{1}{\Psi^{\prime \prime}\left(y_{1}\right)\left(y_{1}-x_{1}\right)} .
\end{gathered}
$$

These derivatives exist everywhere in $B_{\sigma}(x)$ by $(71)$. From (72) we get

$$
\frac{\partial y_{2}}{\partial x_{k}}=\Psi^{\prime}\left(y_{1}\right) \frac{\partial y_{1}}{\partial x_{k}}, \quad k=1,2 .
$$

Thus we obtain

$$
J_{1} g(x)=|D g(x)|=\sqrt{\sum_{i, j=1}^{2}\left(\frac{\partial y_{i}}{\partial x_{j}}\right)^{2}}=\frac{\left[1+\left(\Psi^{\prime}\left(y_{1}\right)\right)^{2}\right]^{\frac{3}{2}}}{\rho \Psi^{\prime \prime}\left(y_{1}\right)},
$$

where $y_{1}=g_{1}(x)$, and $\rho$ is given by (74). From (71), (75) we see that $g$ is Lipschitz on each subdomain

$$
D_{\varepsilon}^{1}=\left\{x \in D^{1} \mid \rho(x)>\varepsilon\right\},
$$

where $\varepsilon>0$.

We can rewrite $(75)$ in the form

$$
J_{1} g(x)=J_{1} g(y, \rho)=\frac{1}{\rho \kappa(y)},
$$


where $\kappa(y)$ is the curvature of $T_{1}$ at $y$.

We have

$$
D u D \varphi=-\frac{\partial \varphi}{\partial \rho} \quad \text { in } \quad D^{1} .
$$

Let the map $\Phi: D^{1} \rightarrow\left\{y \in T_{1}, \rho \in(0, r(y))\right\}$ be defined by $x \rightarrow(y, \rho)$. The map $\Phi$ is one-to-one, onto. Let $\varepsilon>0$. Applying Theorem 3.2.22 of [Fed69] and using (60), (70), (76) we get:

$$
\begin{aligned}
\int_{D_{\varepsilon}^{1}} a_{\mathrm{ac}} D u D \varphi d x & =\frac{f_{0}(t)}{2|D(t)|} \int_{T_{1}}\left\{\int_{\varepsilon}^{r(y)}\left[\left(a_{\mathrm{ac}} D u D \varphi\right) \circ \Phi^{-1}(y, \rho)\right]\left[J_{1} g(y, \rho)\right]^{-1} d \rho\right\} d \mathcal{H}^{1}(y) \\
& =\frac{f_{0}(t)}{2|D(t)|} \int_{T_{1}}\left\{\int_{\varepsilon}^{r(y)} \frac{1}{\rho}\left[r^{2}(y)-\rho^{2}\right]\left(-\frac{\partial \varphi}{\partial \rho}\right) \rho \kappa(y) d \rho\right\} d \mathcal{H}^{1}(y) \\
& =\frac{f_{0}(t)}{2|D(t)|} \int_{T_{1}}\left[\varphi\left(y_{\varepsilon}\right)\left[r^{2}(y)-\varepsilon^{2}\right]-\int_{\varepsilon}^{r(y)} 2 \rho \varphi\left(\Phi^{-1}(y, \rho)\right) d \rho\right] \kappa(y) d \mathcal{H}^{1}(y) \\
& =\frac{f_{0}(t)}{2|D(t)|}\left[\int_{T_{1}} \varphi\left(y_{\varepsilon}\right)\left[r^{2}(y)-\varepsilon^{2}\right] \kappa(y) d \mathcal{H}^{1}(y)-2 \int_{D_{\varepsilon}^{1}} \varphi d x\right],
\end{aligned}
$$

where $y_{\varepsilon}=y+\frac{\varepsilon}{\left|R_{y}\right|}\left[\hat{l}\left(R_{y}\right)-y\right]$. Here $R_{y}$ is the transport ray which has the upper end at $y$. Since $y \in T_{1}$, such $R_{y}$ exists and is unique. Passing to the limit as $\varepsilon \rightarrow 0$ in (78), we get

$$
\int_{D^{1}} a_{\mathrm{ac}} D u D \varphi d x=\frac{f_{0}(t)}{2|D(t)|}\left[\int_{T_{1}} \varphi(y) r^{2}(y) \kappa(y) d \mathcal{H}^{1}(y)-2 \int_{D^{1}} \varphi d x\right] .
$$

The domain $D^{2}$ is considered similarly. Thus, from (68), (79) and the corresponding formula for $D^{2}$ we get:

$$
\begin{aligned}
& \frac{f_{0}(t)}{|D(t)|} \int_{D(t)}[\varphi(O)-\varphi] d x-\int_{D(t)} a_{\mathrm{ac}} D u D \varphi d x \\
& \quad=f_{0}(t) \frac{\left|D^{1}\right|+\left|D^{2}\right|}{|D(t)|} \varphi(O)-\frac{f_{0}(t)}{2|D(t)|} \sum_{i=1}^{2} \int_{T_{i}} \varphi(y) r^{2}(y) \kappa(y) d \mathcal{H}^{1}(y) .
\end{aligned}
$$

Remark 15 At this point we can prove (64). In the domain $D^{3}$ we have $u(x, t)=z(t)-$ $|x-O|$ and $\rho=|x-O|$. Thus we obtain (64) in $D^{3}$ by explicit differentiation. In $D^{1}$ we use (69), (70) and (76) in the following argument. Let $\varphi \in C_{0}^{\infty}\left(D^{1}\right)$. Then we have (77). Thus

$$
\begin{aligned}
& \int_{D^{1}} \varphi \Delta u d x=-\int_{D^{1}} D \varphi D u d x=\int_{T^{1}}\left\{\int_{0}^{r(y)} \frac{\partial \varphi}{\partial \rho} \kappa(y) \rho d \rho\right\} d \mathcal{H}^{1}(y) \\
& =-\int_{T^{1}}\left\{\int_{0}^{r(y)} \varphi d \rho\right\} \kappa(y) d \mathcal{H}^{1}(y)=-\int \frac{\varphi}{\rho} d x .
\end{aligned}
$$

This is true for any $\varphi \in C_{0}^{\infty}\left(D^{1}\right)$. Thus (64) holds in $D^{1}$. Similarly (64) holds in $D^{2}$.

Next we prove that (80) holds for all $\varphi$ satisfying

$$
\varphi \in C^{1}(\Lambda \backslash \mathcal{R}), \quad|D \varphi| \leq C \quad \text { on } \quad \Lambda \backslash \mathcal{R} .
$$


To see this we note that $\varphi$ satisfying (81) is locally bounded on $\Lambda$ and thus define for $\varepsilon>0$ the function $\varphi_{\varepsilon} \in C^{1}(\Lambda)$ such that $\varphi_{\varepsilon} \equiv \varphi$ on $\Lambda \backslash N_{\varepsilon}(\mathcal{R})$ and $\left|D \varphi_{\varepsilon}\right| \leq \frac{C}{\varepsilon}$ on $N_{\varepsilon}(\mathcal{R}) \cap B_{2 R}(O)$, where $R$ is large such that $D(t) \subset B_{R}(O)$. Here $N_{\varepsilon}(\cdot)$ denotes $\varepsilon$ neighborhood of a set in $\mathbf{R}^{2}$. For $\varphi_{\varepsilon}$ the equality (80) holds, and we conclude the proof passing to the limit as $\varepsilon \rightarrow 0$, using (46) and the inequality

$$
\left|a_{\mathrm{ac}}(x)\right| \leq C\left|x-\hat{l}\left(R_{x}\right)\right|
$$

The inequality (82) follows from (60).

Note that $\varphi$ which satisfies (81) can be discontinuous across $\mathcal{R}$.

Similarly, using Lemma 10 instead of (82), we show that (43) holds for all $\varphi$ satisfying (81).

Let

$$
b=\tilde{a}-a_{\mathrm{ac}} d x .
$$

Then $b$ is a signed Radon measure. By (83), (80), (43) we have for all $\varphi$ satisfying (81)

$$
\int_{D(t)} D u D \varphi d b=f_{0}(t) \frac{\left|D^{1}\right|+\left|D^{2}\right|}{|D(t)|} \varphi(O)-\frac{f_{0}(t)}{2|D(t)|} \sum_{i=1}^{2} \int_{T_{i}} \varphi(y) r^{2}(y) \kappa(y) d \mathcal{H}^{1}(y) .
$$

Now we are ready to prove (67), which can be rewritten as

$$
\operatorname{supp}(b) \subset \bar{D}_{\text {sing }} .
$$

Let $\psi$ be a smooth function on $\Lambda$ and $\operatorname{supp}(\psi) \subset D(t) \backslash \bar{D}_{\text {sing }}$. In particular

$$
\psi \equiv 0 \quad \text { in } B_{\sigma}(O) \quad \text { for some } \quad \sigma>0 \text {. }
$$

For $x \in \Lambda \backslash \mathcal{R}$ we denote $P(x)$ the unique shortest path in $\Lambda$ connecting $x$ and $O$. Then $P(x)$ is a $C^{1}$ curve as we have showed above. Define function $\varphi$ on $\Lambda \backslash \mathcal{R}$ by

$$
\varphi(x)=\int_{P(x)} \psi d \mathcal{H}^{1} .
$$

From the structure of sets $P(x)$ and from $(27)$ and (86) it follows that the function (87) satisfies (81). We have

$$
P(x) \subset D_{\text {sing }} \quad \text { for } x \in D_{\text {sing }} \text {. }
$$

Thus

$$
\varphi \equiv 0 \quad \text { on } \quad D_{\text {sing }}
$$

It follows from the definition of $\varphi$ that

$$
D u D \varphi=-\psi \quad \text { on } \quad D(t) \backslash\left(\partial^{\text {int }} D(t) \cup \partial \Lambda\right) .
$$

Now using (84), (88), (89) we get

$$
\int_{\Lambda} \psi d b=-\int_{D(t)} D u D \varphi d b=0
$$

Thus (85) is proved.

Now we prove that $b$ is a nonnegative Radon measure. Let

$$
\psi \in C^{1}(\Lambda), \quad \psi \geq 0, \quad \operatorname{supp}(\psi) \subset \Lambda \backslash B_{\sigma}(O) \text { where } \sigma>0 .
$$


Define the function $\varphi$ on $\Lambda \backslash \mathcal{R}$ by (87). Then, as before, $\varphi$ satisfies (81). From (90) and (87) we have

$$
\varphi(O)=0 \leq \varphi(x), \quad x \in \Lambda \backslash \mathcal{R} .
$$

Now from (89), (84) and convexity of $\Omega$ we get

$$
\int_{D(t)} \psi d b=-\int_{D(t)} D u D \varphi d b=\frac{f_{0}(t)}{2|D(t)|} \sum_{i=1}^{2} \int_{T_{i}} \varphi(y) r^{2}(y) \kappa(y) d \mathcal{H}^{1}(y) \geq 0 .
$$

Using assumption (a) of Definition 7 we conclude that $b$ is nonnegative.

It follows from (85), (84) that for any

$$
\varphi \in C\left(D_{\text {sing }}\right) \cap C^{1}\left(D_{\text {sing }} \backslash\{O\}\right) \quad \text { such that }|D \varphi|<C \text { on } D_{\text {sing }} \backslash\{O\}
$$

we have

$$
\int_{D_{\text {sing }}} D u D \varphi d b=f_{0}(t) \frac{\left|D^{1}\right|+\left|D^{2}\right|}{|D(t)|} \varphi(O)-\frac{f_{0}(t)}{2|D(t)|} \sum_{i=1}^{2} \int_{T_{i}} \varphi(y) r^{2}(y) \kappa(y) d \mathcal{H}^{1}(y) .
$$

Note that $D_{\text {sing }} \backslash\{O\}$ is the union of two disjoint $C^{1}$ curves. If $T_{1}$ and $T_{2}$ have a common endpoint $z$ (and then $\{z\}=\mathcal{R} \cap \partial \Omega$ ), then $z \notin D_{\text {sing }} \backslash\{O\}$ and thus the function $\varphi$ can have jump across the point $z$. On Figure 4 the point $z$ is $Q$, and the function satisfying (91) should be: continuous on $D_{\text {sing }}=O A \cup A Q \cup O B \cup B Q$ (note that this set does not include the point $Q$ ), $C^{1}$-smooth on $O A \cup A Q$ and on $O B \cup B Q$, and have bounded first derivative on $O A \cup A Q \cup O B \cup B Q$ (again, points $O$ and $Q$ are not included).

To prove the uniqueness of mass transport density it is enough to show that $b=a_{\mathrm{S}}$ where $a_{\mathrm{S}}$ is defined by (62).

We prove first that $b$ is absolutely continuous with respect to the 1-dimensional Hausdorff measure on $D_{\text {sing }}$. Let $x \in D_{\text {sing }} \backslash\{O\}$ and $\tilde{T}=D_{\text {sing }} \cap B_{\varepsilon}(x)$, where $B_{\varepsilon}(x)$ is the disk in $\mathbf{R}^{2}$ with the center at $x$ and radius $\varepsilon>0$. If $\varepsilon$ is small enough then $\tilde{T}$ is a connected open arc and $O \notin B_{2 \varepsilon}(x)$. Let $\psi_{\varepsilon} \in C\left(D_{\text {sing }}\right)$ such that $\psi_{\varepsilon} \equiv 1$ on $\tilde{T}, \psi_{\varepsilon} \equiv 0$ on $D_{\text {sing }} \backslash B_{2 \varepsilon}(x)$,

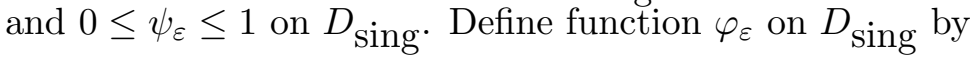

$$
\varphi_{\varepsilon}(x)=\int_{P(x)} \psi_{\varepsilon} d \mathcal{H}^{1}, \quad x \in D_{\text {sing }} .
$$

Then $\varphi_{\varepsilon}$ satisfies (91) and

$$
0 \leq \varphi_{\varepsilon} \leq C \varepsilon, \quad \psi_{\varepsilon}=-D u D \varphi_{\varepsilon} \quad \text { on } \quad D_{\text {sing }} \backslash\{O\} .
$$

We also have $\varphi_{\varepsilon}(O)=0$. Since $b$ is nonnegative, we get from (92)

$$
0 \leq \int_{\tilde{T}} d b \leq \int_{D_{\text {sing }}} \psi_{\varepsilon} d b=-\int_{D_{\text {sing }}} D u D \varphi_{\varepsilon} d b=\frac{f_{0}(t)}{2|D(t)|} \sum_{i=1}^{2} \int_{T_{i}} \varphi_{\varepsilon} r^{2} \kappa d \mathcal{H}^{1} \leq C \frac{f_{0}(t)}{|D(t)|} \varepsilon
$$

where $C$ depends only on $z(t), \mathcal{H}^{1}(\partial \Omega)$ and $\max \kappa$. Since $D_{\text {sing }} \backslash\{O\}$ is the union of two disjoint $C^{1}$ curves, it follows that $b$ is absolutely continuous with respect to the 1-dimensional Hausdorff measure on $D_{\text {sing }}$ and that

$$
b=\tilde{m} \mathcal{H}^{1} \quad \text { on } \quad D_{\text {sing }},
$$


where $\tilde{m}$ is a $\mathcal{H}^{1}$-measurable function on $D_{\text {sing }}$. By (93) the function $\tilde{m}$ is bounded. Thus (92) holds for $\varphi \in \operatorname{Lip}\left(D_{\text {sing }}\right)$.

We will find $\tilde{m}$ from the equation (92) using (94). Let first $z \in R_{\tan }^{1} \backslash\{O\}$. Let $\varepsilon>0$ be small such that $\operatorname{dist}(O, z)>\varepsilon$. Define

$$
\begin{aligned}
& \varphi_{\varepsilon}(x)= \begin{cases}0 & \text { if } d_{\Lambda}(x, O)-d_{\Lambda}(z, O)<-\frac{\varepsilon}{2}, \\
\frac{1}{2}+\frac{1}{\varepsilon}\left[d_{\Lambda}(x, O)-d_{\Lambda}(z, O)\right] & \text { if }\left|d_{\Lambda}(x, O)-d_{\Lambda}(z, O)\right| \leq \frac{\varepsilon}{2}, \\
1 & \text { if } d_{\Lambda}(x, O)-d_{\Lambda}(z, O)>\frac{\varepsilon}{2},\end{cases} \\
& \quad \text { for } x \in R_{\tan }^{1} \cup T_{1} ; \\
& \varphi_{\varepsilon}(x)=0, \quad \text { for } x \in R_{\tan }^{2} \cup T_{2} .
\end{aligned}
$$

Then $\varphi_{\varepsilon} \in \operatorname{Lip}\left(D_{\text {sing }}\right)$. We have on $R_{\tan }^{1} \cup T_{1}$

$$
D u D \varphi_{\varepsilon}= \begin{cases}-\frac{1}{\varepsilon} & \text { if } \quad\left|d_{\Lambda}(x, O)-d_{\Lambda}(z, O)\right|<\frac{\varepsilon}{2}, \\ 0 & \text { if } \quad\left|d_{\Lambda}(x, O)-d_{\Lambda}(z, O)\right|>\frac{\varepsilon}{2} .\end{cases}
$$

Note that

$$
\begin{aligned}
\varphi_{\varepsilon}(O) & =0, \\
\varphi_{\varepsilon} & \equiv 1 \text { on } T_{1},
\end{aligned}
$$

for small $\varepsilon$. Inserting $\varphi_{\varepsilon}$ instead of $\varphi$ into the equality (92), using (94) and passing to the limit as $\varepsilon \rightarrow 0$ in the resulting equality, we obtain for $\mathcal{H}^{1}$ a.e. $z \in R_{\tan }^{1}$

$$
\tilde{m}(z)=\frac{f_{0}(t)}{2|D(t)|} \int_{T_{1}} r^{2}(y) \kappa(y) d \mathcal{H}^{1}(y) .
$$

It remains to show that the integral in right-hand side of (97) equals to $2\left|D^{1}\right|$. We show this by changing variables $(y, \rho) \rightarrow x$ in $D^{1}$, where $(y, \rho)$ defined by $(69)$ and using the Jacobian formula (76):

$$
\int_{T_{1}} r^{2}(y) \kappa(y) d \mathcal{H}^{1}(y)=\int_{T_{1}}\left[\int_{0}^{r(y)} 2 \rho d \rho\right] \kappa(y) d \mathcal{H}^{1}(y)=2 \int_{D^{1}} d x=2\left|D^{1}\right| .
$$

The case $z \in R_{\mathrm{tan}}^{2}$ is similar. Thus we proved that

$$
\tilde{m}(z)=f_{0}(t) \frac{\left|D^{k}\right|}{|D(t)|} \quad \text { for a. e. } \quad z \in R_{\text {tan }}^{k}, \quad k=1,2 .
$$

By Remark 14 (1), the (99) is in fact (62) for $z \in R_{\tan }^{k} \backslash\{O\}, k=1,2$.

Now let $z$ be a point in the relative interior of $T_{1}$. Define the function $\varphi_{\varepsilon} \in \operatorname{Lip}\left(D_{\operatorname{sing}}\right)$ by (95). Then we have (96). Now

$$
\begin{array}{lll}
\varphi_{\varepsilon} \equiv 1 & \text { on } \quad T(z) \backslash B_{\varepsilon}(z), \\
\varphi_{\varepsilon} \equiv 0 & \text { on } \quad D_{\text {sing }} \backslash\left(T(z) \cup B_{\varepsilon}(z)\right)
\end{array}
$$

for small $\varepsilon$, where $T(z)$ is defined by $(38)$ and $B_{\varepsilon}(z)$ is the disk in $\mathbf{R}^{2}$ with the center $z$ and radius $\varepsilon$. Now inserting $\varphi_{\varepsilon}$ into the equality (92), using (94), and taking limit as $\varepsilon \rightarrow 0$ in the resulting equality, we obtain for $\mathcal{H}^{1}$ a.e. $z \in T_{1}$

$$
\tilde{m}(z)=\frac{f_{0}(t)}{2|D(t)|} \int_{T(z)} r^{2}(y) \kappa(y) d \mathcal{H}^{1}(y)
$$


Calculating the integral in the right-hand side of the last equality similar to (98) and using (37), we get

$$
\int_{T(z)} r^{2}(y) \kappa(y) d \mathcal{H}^{1}(y)=\int_{T(z)}\left[\int_{0}^{r(y)} 2 \rho d \rho\right] \kappa(y) d \mathcal{H}^{1}(y)=2 \int_{S(z)} d x=2|S(z)| .
$$

The case $z \in T_{2}$ is similar.

Thus we proved that $\tilde{m}$ is defined by $(62)$ a. e. on $D_{\text {sing. Thus the uniqueness of mass }}$ transport density is proved.

In order to prove the existence of mass transport density, we need only to verify that the measure $a$ defined in the formulation of Theorem 11 satisfies the equation (43) for all smooth $\varphi$. From (80), it is enough to prove that we have

$$
\int_{D_{\text {sing }}} D u D \varphi d a_{\mathrm{S}}=f_{0}(t) \frac{\left|D^{1}\right|+\left|D^{2}\right|}{|D(t)|} \varphi(O)-\frac{f_{0}(t)}{2|D(t)|} \sum_{i=1}^{2} \int_{T_{i}} \varphi(y) r^{2}(y) \kappa(y) d \mathcal{H}^{1}(y)
$$

for any $\varphi \in C^{1}\left(D_{\text {sing }}\right)$, where $a_{\mathrm{S}}$ is defined by $(62)$.

Introduce the variable $s$ on the $C^{1}$ curve $T_{1} \cup R_{\tan }^{1}$ by $s=L(x)$ for $x \in T_{1} \cup R_{\text {tan }}^{1}$, where $L(x)$ is the length of the part of the curve between $O$ and $x$. Denote

$$
M_{1}=\left|R_{\tan }^{1}\right|, \quad M_{2}=\mathcal{H}^{1}\left(T_{1}\right) .
$$

We have $L(x)=d_{\Lambda}(x, O)$ on $T_{1} \cup R_{\text {tan }}^{1}$, and $L$ is one-to-one mapping from $T_{1} \cup R_{\text {tan }}^{1}$ onto $\left[0, M_{1}+M_{2}\right)$. In addition we have $T(x)=L^{-1}\left(I_{x}\right)$, where $I_{x}=\left[L(x), M_{1}+M_{2}\right)$. Here $T(x)$ is the set (38). Then by (62), (100) the density of the measure $a_{\mathrm{S}}$ on $T_{1} \cup R_{\mathrm{tan}}^{1}$ satisfies:

$$
\begin{aligned}
m\left(L^{-1}(s)\right) & \equiv f_{0}(t) \frac{\left|D^{1}\right|}{|D(t)|} \quad \text { for } \quad s \in\left(0, M_{1}\right] \\
m\left(L^{-1}(s)\right)=\frac{f_{0}(t)}{2|D(t)|} \int_{s}^{M_{1}+M_{2}} r^{2}\left(L^{-1}(\eta)\right) \kappa\left(L^{-1}(\eta)\right) d \eta & \text { for } \quad s \in\left(M_{1}, M_{1}+M_{2}\right) .
\end{aligned}
$$

In particular,

$$
\lim _{s \rightarrow\left(M_{1}+M_{2}\right)-} m\left(L^{-1}(s)\right)=0 .
$$

Since $R_{\mathrm{tan}}^{1}$ is tangent to $T_{1}$ at their contact point, it follows that $L^{-1}:\left[0, M_{1}+M_{2}\right) \rightarrow \mathbf{R}^{2}$ is a $C^{1}$ mapping. Then for $\varphi \in C^{1}\left(D_{\text {sing }}\right)$ we have

$$
\operatorname{DuD} \varphi(x)=-\left.\frac{d\left(\varphi\left(L^{-1}(s)\right)\right)}{d s}\right|_{s=L(x)} \quad \text { on } T^{1} \cup R_{\mathrm{tan}}^{1}
$$

since $|D u|$ is the unit vector, tangential to the curve $T^{1} \cup R_{\text {tan }}^{1}$. Thus we calculate using $(102)-(104)$ :

$$
\begin{aligned}
\int_{T^{1} \cup R_{\tan }^{1}} D u D \varphi d a_{s} & =\int_{T^{1} \cup R_{\tan }^{1}} D u D \varphi m d \mathcal{H}^{1} \\
& =-\int_{0}^{M_{1}+M_{2}} \frac{d\left(\varphi \circ L^{-1}\right)}{d s}\left(m \circ L^{-1}\right) d s
\end{aligned}
$$




$$
\begin{aligned}
= & f_{0}(t) \varphi(O) \frac{\left|D^{1}\right|}{|D(t)|}+\int_{M_{1}}^{M_{1}+M_{2}}\left(\varphi \circ L^{-1}\right) \frac{d\left(m \circ L^{-1}\right)}{d s} d s \\
= & f_{0}(t) \varphi(O) \frac{\left|D^{1}\right|}{|D(t)|} \\
& \quad-\frac{f_{0}(t)}{2|D(t)|} \int_{M_{1}}^{M_{1}+M_{2}} \varphi\left(L^{-1}(s)\right) r^{2}\left(L^{-1}(s)\right) \kappa\left(L^{-1}(s)\right) d s \\
= & f_{0}(t) \varphi(O) \frac{\left|D^{1}\right|}{|D(t)|}-\frac{f_{0}(t)}{2|D(t)|} \int_{T_{1}} \varphi r^{2} \kappa d \mathcal{H}^{1} .
\end{aligned}
$$

This and a similar calculation for $T_{2} \cup R_{\text {tan }}^{2}$ imply (101).

Theorem 11 is proved.

In the light of Theorem 11 the geometry of mass transfer for the single point source solution can be described as following. Incoming sand moves "downhill" along transport rays and fills domains $D^{1}, D^{2}, D^{3}$. The absolutely continuous part of the mass transport density describes this process, i.e., gives the rate of mass transport at each interior point. In addition sand should be transported from the source to the upper ends of the transport rays that lie in the domains $D^{1}$ and $D^{2}$. The singular part $a_{s}$ of the mass transport density describes this process. The structure of the support of $a_{s}$ and the mass balance at each point of the support of $a_{s}$ given by the equation (62) show the following. In order to reach the domain $D^{1}$ a sand particle moves from the source along the tangent to the obstacle ray $R_{\text {tan }}^{1}$ towards the boundary of the obstacle, then moves along the boundary until it reaches the upper end of its transport ray, and slides down along the transport ray. For $D^{2}$ the picture is similar. Thus each particle moves to its destination point along the shortest path.

Acknowledgement. I would like to thank L. C. Evans for many stimulating discussions. Part of this work was done at MSRI, and I gratefully acknowledge support and excellent research environment. I would like to thank the referee for very helpful remarks. The research is supported in part by National Science Foundation Grant DMS-9623276.

\section{References}

[Ar72] G. Aronsson. A mathematical model in sand mechanics: Presentation and analysis, SIAM J. Appl. Math. 22 (1972), 437-458.

[AEW96] G. Aronsson, L. C. Evans, Y. Wu. Fast/Slow diffusion and growing sandpiles, J.Diff.Equat., 131 (1996), no. 2, 304-335.

[E] L. C. Evans. Partial Differential Equations and Monge-Kantorovich mass transfer. Preprint.

[EFG97] L. C. Evans, M. Feldman, R. F. Gariepy. Fast/Slow diffusion and collapsing sandpiles, J.Diff.Equat., 137(1997), 166-209.

[EGan] L. C. Evans, W. Gangbo. Differential equations methods for the Monge-Kantorovich mass transfer problem, Preprint.

[Fed69] H. Federer. Geometric measure theory, Springer, 1969.

[F97] M. Feldman. Variational evolution problems and nonlocal geometric motion, MSRI Preprint No. 1997-098, 1997.

[J96] U. Janfalk. On a minimization problem for vector fields in $L^{1}$, Bull. London Math. Soc. 28 (1996), no. 2, 165-176.

[P96] L. Prigozhin. Variational model of sandpile growth, European J. Appl. Math. 7 (1996), no. $3,225-235$. 
[R84] S. T. Rachev. The Monge-Kantorovich mass transference problem and its stochastic applications, Theory Prob. Appl., 29(1984), 647-676. 